\title{
MAXIMAL SEPARATION THEOREMS FOR CONVEX SETS
}

\author{
BY \\ VICTOR KLEE( $\left.{ }^{1}\right)$
}

Introduction. Throughout the paper $E$ denotes a finite-dimensional Euclidean space. Attention is restricted to subsets of $E$ though some of the results have infinite-dimensional analogues. Separation theorems for convex sets are basic in the theory of convexity and in the applications of convexity to other parts of mathematics. The standard separation theorem asserts that any two nonempty disjoint convex subsets of $E$ are separated by a hyperplane; this involves a very weak type of separation. Other well-known separation theorems deal with compact or open convex sets and with rather strong types of separation. Several additional separation theorems have appeared in the literature [2], [4], [6], most of them directed at classes of closed convex sets (intersections of closed halfspaces). Such theorems are of interest in connection with systems of weak ( $\leqq)$ linear inequalities. The main separation theorems of this paper are directed at classes of evenly convex sets (intersections of open halfspaces [1]) and are thus of interest in connection with systems of strong (<) linear inequalities or mixed systems of inequalities. Rockafellar's separation theorem [10] for partially polyhedral sets is extended to a wider class of sets. The attempt to obtain separation theorems under minimal hypotheses leads to the notion of a maximal separation theorem. Eighteen maximal theorems are presented here, involving four different types of separation.

Definitions and preliminaries. Let us begin by defining the various types of separation to be considered. A set $X$ is said to be separated from a set $Y$ by a hyperplane $H$ provided that $X$ lies in one of the closed halfspaces bounded by $H$ and $Y$ lies in the other. In contrast to the other types of separation, this does not require disjointness of $X$ and $Y$; however, we shall be concerned only with disjoint sets. The set $X$ is nicely separated from $Y$ by $H(X \mid Y$ by $H)$ provided that the separating hyperplane $H$ is disjoint from $X$ or from $Y$ (without specifying which), openly separated from $Y$ by $H(X \cdot \mid Y$ by $H)$ provided that $H$ is disjoint from $X$, and closedly separated from $Y$ by $H(X \mid \cdot Y$ by $H)$ provided that $H$ is disjoint from $Y$. Thus $X$ is openly or closedly separated from $Y$ by $H$ according as $X$ lies in an open or a closed halfspace disjoint from $Y$ and bounded by $H$. The set $X$ is strictly separated from $Y$ by $H(X \cdot \mid \cdot Y$ by $H)$ provided that the separating hyperplane $H$ is

Received by the editors May 31, 1967.

(1) This study was supported in part by the RAND Corporation and in part by the National Science Foundation (NSF-GP-3579). 
disjoint from both $X$ and $Y$, and strongly separated from $Y$ by $H(X \cdot \| \cdot Y$ by $H)$ provided that $H$ is at positive distance from both $X$ and $Y$. The same terms will be applied to any linear form $f$ whose level sets are hyperplanes parallel to $H$. If the sets $X$ and $Y$ are separated by the linear form $f$, or if $X$ and $Y$ are convex, then the various stronger types of separation are equivalent to the following conditions on the subsets $f X$ and $f Y$ of the real line $R$ :

$$
\begin{array}{lll}
X \mid Y & \text { by } f & (f X) \cap(f Y)=\varnothing, \\
X \cdot \mid Y & \text { by } f & (f X) \cap(\operatorname{cl} f Y)=\varnothing, \\
X \mid \cdot Y & \text { by } f & (\operatorname{cl} f X) \cap(f Y)=\varnothing, \\
X \cdot \mid \cdot Y & \text { by } f & (f X) \cap(\operatorname{cl} f Y) \cup(\operatorname{cl} f X) \cap(f Y)=\varnothing, \\
X \cdot \| \cdot Y & \text { by } f & (\operatorname{cl} f X) \cap(\operatorname{cl} f Y)=\varnothing .
\end{array}
$$

Henceforth, $\sigma$ will denote any of the separation relations just defined and $\rho$ will denote the inverse of $\sigma$, so that $\sigma$ and $o$ are the same except when one represents $\cdot \mid$ and the other $\mid \cdot$. The statement $X \sigma Y$ will mean that there exists a hyperplane $H$ such that $X \sigma Y$ by $H$. Note that

$$
X|Y \Leftrightarrow X \cdot| Y \text { or } X \mid \cdot Y
$$

and

$$
X \cdot|\cdot Y \Leftrightarrow X \cdot| Y \text { and } X \mid \cdot Y .
$$

(If $X \cdot \mid Y$ there is a linear form $f$ such that $f(x)<\inf f Y$ for all $x \in X$. If $X \mid \cdot Y$ there is a linear form $g$ such that $\sup g X<f(y)$ for all $y \in Y$. With $\mu=\sup g X+\inf f Y$, we have

$$
(f+g)(x)<\mu<(f+g)(y) \text { for all } x \in X, y \in Y
$$

and consequently $X \cdot \mid \cdot Y$ by $f+g$.)

By the standard separation theorem, $X$ is separated from $Y$ whenever $X$ and $Y$ are disjoint nonempty convex subsets of $E$. However, when $\operatorname{dim} E \geqq 2$ it is not necessary that $X \mid Y$. For example, let $H$ be a hyperplane in $E, A$ and $B$ the two open halfspaces bounded by $H$, and $a$ and $b$ distinct points of $H$. With $X=A \cup\{a\}$ and $Y=B \cup\{b\}$, the convex sets $X$ and $Y$ meet every hyperplane which separates them. If two disjoint nonempty convex subsets $X$ and $Y$ of $E$ are closed, then $X \mid Y$ when $\operatorname{dim} E \leqq 2$ but not necessarily when $\operatorname{dim} E \geqq 3$. For example, let

$$
X=\{(0, \beta, 1): \beta \in R\}
$$

and

$$
Y=\left\{(\alpha, \beta, \gamma): \alpha \beta \geqq \gamma^{2}, \alpha \geqq 0, \beta \geqq 0, \gamma \geqq 0\right\},
$$

disjoint nonempty closed convex subsets of $R^{3}$ not nicely separated by any hyperplane. (The example is due to T. A. Botts [3, p. 459]. The same idea will be used below in a general construction.) Distinctions among the other types of separation are easily illustrated by closed convex subsets of $R^{2}$. 
For classes $\mathscr{X}$ and $\mathscr{Y}$ of convex sets, $\mathscr{X} \sigma \mathscr{Y}$ will mean that $X \sigma Y$ whenever $X$ and $Y$ are disjoint nonempty members of $\mathscr{X}$ and $\mathscr{Y}$ respectively. An ordered pair $(\mathscr{X}, \mathscr{Y})$ will be called a $\sigma$-pair provided that the assertion $\mathscr{X} \sigma \mathscr{Y}$ is true and a maximal $\sigma$-pair provided that, in addition, the assertion $\mathscr{X} \sigma \mathscr{Y}$ becomes false if either $\mathscr{X}$ or $\mathscr{Y}$ is replaced by a class of convex sets (in the ambient space $E$ ) properly containing it; in the second instance, the assertion $\mathscr{X} \sigma \mathscr{Y}$ will be called a maximal separation theorem or a maximal theorem for $\sigma$. In a systematic study of separation properties it seems natural to seek maximal theorems, for each one is in a sense a best possible result. Further, a maximal theorem $\mathscr{X} \sigma \mathscr{Y}$ yields some insight into the structure of the individual members of $\mathscr{X}$ and $\mathscr{Y}$, for the membership of a set in $\mathscr{X}$ is characterized by its $\sigma$-separability from all disjoint members of $\mathscr{Y}$ and membership in $\mathscr{Y}$ is characterized by $\boldsymbol{o}$-separability from all disjoint members of $\mathscr{X}$.

Since any two disjoint nonempty convex subsets of $E$ are separated, it is plain there is only one maximal theorem for ordinary separation.

It follows from the maximality principle that for any two classes $\mathscr{X}$ and $\mathscr{Y}$ of convex sets such that $\mathscr{X} \mathscr{Y}^{\prime}$ there is at least one maximal $\sigma$-pair $\left(\mathscr{X}^{\prime}, \mathscr{Y}^{\prime}\right)$ for which $\mathscr{X}^{\prime} \supset \mathscr{X}$ and $\mathscr{Y}^{\prime} \supset \mathscr{Y}$. However, the maximal theorem $\mathscr{X}^{\prime} \sigma \mathscr{Y}^{\prime}$ is of no interest unless the classes $\mathscr{X}^{\prime}$ and $\mathscr{Y}^{\prime}$ are of some intrinsic interest. For any class $\mathscr{X}$ of convex subsets of $E$ let $\mathscr{X}^{\sigma}$ denote the class of all convex subsets $Y$ of $E$ such that $X \sigma Y$ whenever $X \in \mathscr{X}$ and $X \cap Y=\varnothing$. The following result, an immediate consequence of the relevant definitions, exhibits a maximal theorem for $\sigma$ which is associated with $\mathscr{X}$ in a natural way.

THEOREM 1. For any class $\mathscr{X}$ of convex subsets of E, the pair $\left(\left(\mathscr{X}^{\circ}\right)^{\circ}, \mathscr{X}^{\circ}\right)$ is a maximal $\sigma$-pair; it is the only maximal $\sigma$-pair $\left(\mathscr{X}^{\prime}, \mathscr{Y}^{\prime}\right)$ for which $\mathscr{X}^{\prime} \supset \mathscr{X}$ and $\mathscr{Y}^{\prime} \supset \mathscr{X}^{\sigma}$.

For any $\sigma$-pair $(\mathscr{X}, \mathscr{Y})$, the maximal $\sigma$-pairs $\left(\left(\mathscr{X}^{\sigma}\right)^{\rho}, \mathscr{X}^{\sigma}\right)$ and $\left(\mathscr{Y}^{\rho},\left(\mathscr{Y}^{\circ}\right)^{\sigma}\right)$ will be called the maximal $\sigma$-pairs naturally associated with $(\mathscr{X}, \mathscr{Y})$. They are not necessarily distinct.

Maximal theorems for strong separation and strict separation. For any two subsets $X$ and $Y$ of $E, \delta(X, Y)$ will denote the distance between $X$ and $Y$; that is, $\delta(X, Y)=\inf \{\|x-y\|: x \in X, y \in Y\}$. The set $X$ is called an asymptote of $Y$ provided that $X$ is a flat in $E \sim Y$ with $\delta(X, Y)=0$; a $j$-dimensional asymptote is called a $j$-asymptote. (Thus the term asymptote is used as in [6], [7] but not quite as in [2].) Plainly $Y$ admits a $j$-asymptote if and only if $Y$ 's orthogonal projection on some (dim $E-j)$-dimensional flat in $E$ fails to be closed. The sets which admit no 0 -asymptote are exactly the closed sets, and the sets admitting no asymptote whatever are those whose projections (or, equivalently, whose affine images) are all closed. A convex set $Y$ is called continuous [2] provided that $Y$ is closed and its support function (defined on the unit sphere $\{u \in E:\|u\|=1\}$, permitted to assume the values $\pm \infty$ ) is continuous; this is equivalent [2] to saying $Y$ admits no boundary ray and no 1-asymptote, as well as [6] to saying $Y$ admits no boundary ray and no asymptote of any dimension. 
THEOREM 2. For disjoint nonempty convex subsets $X$ and $Y$ of $E$, each of the following conditions implies $X$ is strongly separated from $Y$. Each represents a maximal theorem for $\cdot \| \cdot$.

(a) $X$ is continuous and $Y$ is closed.

(b) Neither $X$ nor $Y$ admits an asymptote.

THEOREM 3. For disjoint nonempty convex subsets $X$ and $Y$ of $E$, each of the following conditions implies $X$ is strictly separated from $Y$. Each represents a maximal theorem for $\cdot 1 \cdot$.

(a) $X$ is continuous and $Y$ is closed.

(b) Neither $X$ nor $Y$ admits an asymptote.

(c) $X$ and $Y$ are both closed and neither has a boundary ray.

(d) $X$ and $Y$ are both open.

Proofs. That $X \cdot \| \cdot Y$ was proved in [2, Theorem 1.3] for (a) and in [6, Theorem 5] for (b). Plainly the same conditions imply $X \cdot \mid \cdot Y$. That $X \cdot \mid \cdot Y$ follows from [4, Theorem 4] for (c) and is well known for (d). To show the separation theorems corresponding to (a) and (b) are maximal for $\cdot \mid \cdot$ (and hence, a fortiori, for $\cdot \| \cdot$ ), we must show that if $C$ is a proper convex subset of $E$ and $C$ is not continuous, or $C$ is not closed, or $C$ admits an asymptote, then $E \sim C$ contains a nonempty convex set $K$ which is not strictly separated from $C$ even though (respectively) $K$ is closed or $K$ is continuous or $K$ admits no asymptote. This is easily accomplished by the constructions described below in connection with open separation. The treatment of (c) and (d) is similarly straightforward.

The strict separation theorems (a), (b) and (c) are all extensions of the assertion that disjoint compact convex sets are strictly separated. The maximal $\sigma$-pairs indicated in (a) and (b) (for $\sigma=\cdot \| \cdot$ or $\sigma=\cdot \mid \cdot$ ) are naturally associated with various well-known $\sigma$-pairs, including the following: (a) ( $X$ onepointed, $Y$ closed) or ( $X$ compact, $Y$ closed); (b) ( $X$ a flat, $Y$ a flat) or ( $X$ a polyhedron, $Y$ a polyhedron).

Maximal theorems for open separation and nice separation. A subset $X$ of $E$ is called evenly convex [1] provided that $X$ is the intersection of a family of open halfspaces or, equivalently, that $X$ is openly separated from every onepointed subset of $E \sim X$. As was remarked by Fenchel [1], this is equivalent to requiring that $X$ is connected and each point of $E \sim X$ lies in a hyperplane disjoint from $X$. Note that all projections (or, equivalently, all affine images) of $X$ are evenly convex if and only if $X$ is connected and every flat in $E \sim X$ lies in a hyperplane disjoint from $X$.

A subset of $E$ is called a strip provided that it is convex, is different from $E$, and is a union of translates of a hyperplane. Equivalently, a strip is a hyperplane, an open or closed halfspace, or a set of the form $S$ or $H_{1} \cup S$ or $H_{1} \cup S \cup H_{2}$, where $H_{1}$ and $H_{2}$ are parallel hyperplanes and $S$ is the set of all points of $E$ lying between $H_{1}$ and $H_{2}$. All strips are evenly convex. 
A hyperplane $H$ is said to support a set $X$ in $E$ provided that $\delta(H, X)=0$ and $X$ lies in one of the closed halfspaces bounded by $H$. The set $X$ is said to be continuous relative to $H$ provided that the intersection $X \cap H$ is closed and convex but admits neither boundary ray nor asymptote relative to $H$. In neither of these definitions is $X$ required to intersect $H$.

A subset of $E$ is called a polytope provided that it is the convex hull of a finite set of points and a polyhedron provided that it is the intersection of a finite family of closed halfspaces. The polytopes are exactly the bounded polyhedra. A subset $X$ of $E$ is said to be boundedly polyhedral provided that its intersection with any polytope is a polytope and to be polyhedral at a point $p$ of $X$ provided that $X$ contains a polytope which is a neighborhood of $p$ relative to $X$. A set is boundedly polyhedral if and only if it is closed, convex, and polyhedral at each of its points [5, 2.17].

THEOREM 4. For disjoint nonempty convex subsets $X$ and $Y$ of $E$, each of the following conditions implies $X$ is openly separated from $Y$. Each represents a maximal theorem for $\cdot \mid$ except that $(\mathrm{j})$ does not when $E$ is two-dimensional.

(e) $X$ is open; $Y$ is arbitrary.

(f) $X$ is evenly convex and its intersection with any supporting hyperplane is compact; $Y$ is closed.

(g) $X$ admits no asymptote in any supporting hyperplane intersecting $X ; Y$ admits no asymptote.

(h) $X$ is evenly convex and its intersection with any supporting hyperplane is closed; $Y$ is evenly convex, $Y$ intersects and is continuous relative to any supporting hyperplane.

(i) X's projections are all evenly convex; $Y$ admits no asymptote and is boundedly polyhedral.

(j) $X$ is evenly convex; $Y$ is onepointed or a closed strip.

THEOREM 5. For disjoint nonempty convex subsets $X$ and $Y$ of $E$, each of the following conditions implies $X$ is nicely separated from $Y$. Each represents a maximal theorem for | except that (q) does not when $E$ is two-dimensional.

(k) $X$ is open or a strip; $Y$ is arbitrary.

(l) $X$ is evenly convex and is continuous relative to any supporting hyperplane; $Y$ is evenly convex and its intersection with any supporting hyperplane is closed.

(m) $X$ admits no asymptote in any supporting hyperplane intersecting $X ; Y$ admits no asymptote in any supporting hyperplane intersecting $Y$.

(n) $X$ is evenly convex and its intersection with any supporting hyperplane is closed; $Y$ is evenly convex and is continuous relative to any supporting hyperplane.

(o) $X$ 's projections are all evenly convex and $X$ is polyhedral at each of its points; $Y$ 's projections are all evenly convex and $Y$ is polyhedral at each of its points.

(p) $X$ 's projections are all evenly convex; $Y$ admits no asymptote in any supporting hyperplane intersecting $Y$ and $Y$ is polyhedral at each of its points.

(q) $X$ is evenly convex; $Y$ is onepointed or open or a strip. 
CoRollary. If $X$ and $Y$ are as described in any of the above conditions then the sets $X+Y$ and $X-Y$ are evenly convex.

Theorems 4 and 5 are proved in the next section. The present section contains a proof of the Corollary and some comments on the theorems.

Proof of the Corollary. Since $X+Y=X-(-Y)$, and since the various conditions on $X$ and $Y$ are all preserved under nonsingular affine transformations, it suffices to consider $X-Y$ in proving the Corollary. Suppose that $X$ and $Y$ are proper convex subsets of $E$, not necessarily disjoint, and that one of the conditions (a)-(q) is satisfied. We want to show that each point $z$ of $E \sim(X-Y)$ lies in a hyperplane disjoint from $X-Y$. Note that the sets $X$ and $Y+z$ are disjoint, for otherwise there exist $x \in X$ and $y \in Y$ such that $x=y+z$ and $z=x-y \in X-Y$. With $X$ and $Y+z$ disjoint, the above theorems imply they are nicely separated. That is, $E$ admits a linear form $f$ such that $f(x)<f(y+z)$ for all $x \in X$ and $y \in Y$, whence

$$
\{p \in E: f(p)=f(z)\}
$$

is a hyperplane which includes $z$ and misses $X-Y$. The proof of the Corollary is complete.

A subset of $E$ is called partially polyhedral [10] provided that it is the intersection of a finite family of strips or, equivalently, that it is the intersection of a finite family of open or closed halfspaces (both sorts of halfspaces being allowed in the family). In his Separation Lemma [10], Rockafellar has proved that if $X$ is a partially polyhedral set in $E$ then every flat in $E \sim X$ lies in a hyperplane in $E \sim X$. Thus $X$ 's projections are all evenly convex. Plainly $X$ is polyhedral at each of its points, and hence the separation theorem represented by condition (o) above extends Rockafellar's theorem [10] asserting that disjoint nonempty partially polyhedral sets are nicely separated.

When $E$ is two-dimensional, conditions (i), (j), (p) and (q) are all equivalent so far as $X$ is concerned, for all one-dimensional convex sets are evenly convex. However, (i) is weaker than ( $\mathrm{j}$ ) and (p) is weaker than (q) so far as $Y$ is concerned, whence it is evident that the $\cdot \mid$-pair indicated by $(j)$ and the $\mid$-pair indicated by (q) are not maximal. As we shall see, this anomaly appears only when $E$ is twodimensional.

The separation theorem represented by $(n)$ is the same as that represented by (l). Theorem 5 is stated in this redundant way to facilitate a comparison with Theorem 4. Note that the various conditions on $X$ and $Y$ are related as shown in Figure 1. Most of the listed implications are obvious, but it may be desirable to show that all projections of a set $W$ are evenly convex if $W$ admits no asymptote in any supporting hyperplane intersecting $W$. Suppose that $\pi$ is the transformation projecting $E$ orthogonally onto a flat $F$ in $E$ and that $\pi W$ is not evenly convex. Then there is a point $z \in F \sim \pi W$ such that $z$ does not lie in any (relative) hyperplane in $F \sim \pi W$. From this it is clear, since $\pi W$ is convex, that $\delta(z, \pi W)=0$ and consequently 


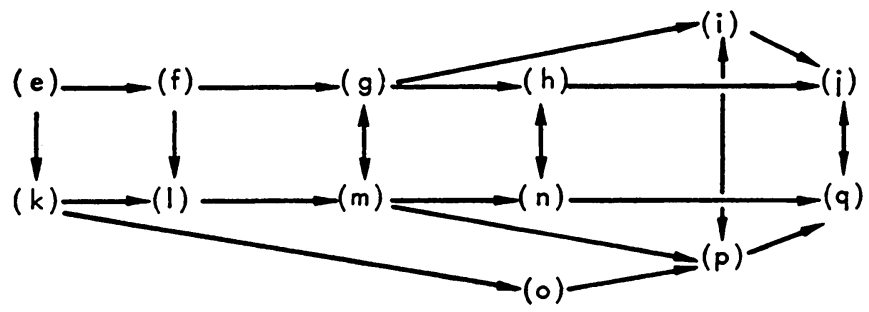

Conditions on $X$

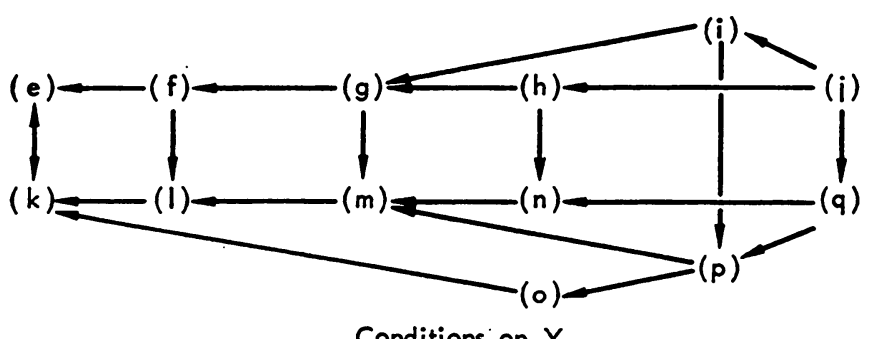

FIGURE 1

$\delta\left(\pi^{-1}\{z\}, W\right)=0$. Clearly $z$ lies in some supporting hyperplane $H$ of $\pi W$ relative to $F$, and then $\pi^{-1} H$ is a supporting hyperplane of $W$ in $E$ containing the asymptote $\pi^{-1}\{z\}$ of $W$.

The maximal $\cdot \mid$-pairs indicated by (e), (f), (g), (h), (i) and (j) are contained termwise in the maximal |-pairs indicated by $(k),(1),(m),(n),(p)$ and $(q)$ respectively. In fact (as follows from the construction in the next section) the latter pair is in each case the unique maximal /-pair naturally associated with the former. Thus $\left(\left(\mathscr{X}^{\prime}\right)^{\prime}, \mathscr{X}^{\prime}\right)=\left(\mathscr{Y}^{\prime},\left(\mathscr{Y}^{\prime}\right)^{\prime}\right)$ for each of the pairs $(\mathscr{X}, \mathscr{Y})$ indicated in Theorem 4 . This equality does not hold for all |-pairs $(\mathscr{X}, \mathscr{Y})$. Indeed, let $\mathscr{X}$ be the class of all compact convex subsets of $E$ and $\mathscr{Y}$ the class of all polyhedra in $E$. Then $\left(\left(\mathscr{X}^{\prime}\right)^{\prime}, \mathscr{X}^{\prime}\right)$ and $\left(\mathscr{Y} \mid,\left(\mathscr{Y}^{\prime}\right)^{\prime}\right)$ are the maximal |-pairs indicated by $(\mathrm{l})$ and $(\mathrm{p})$ respectively. The maximal |-pair indicated by $(\mathrm{m})$ contains the pair $(\mathscr{X}, \mathscr{Y})$ but is not naturally associated with $(\mathscr{X}, \mathscr{Y})$.

Proofs of the theorems on open separation and nice separation. The proofs of the theorems are based on a remark and two lemmas.

REMARK. If $X$ is evenly convex, $p \in X$, and $w \in \mathrm{cl} X$, then $[p, w[\subset X$.

Proof. When $X$ is evenly convex each point $q$ of $E \sim X$ lies in a closed halfspace $Q$ disjoint from $X$. If $q \in[p, w$ [ then $p \in Q$ or $w \in$ int $Q$, a contradiction in either case.

A familiar two-dimensional example (Figure 2) shows that, in general, the even convexity of $X$ is not implied by the property expressed in the Remark. However, the two properties are equivalent when the closure of $X$ is boundedly polyhedral. 


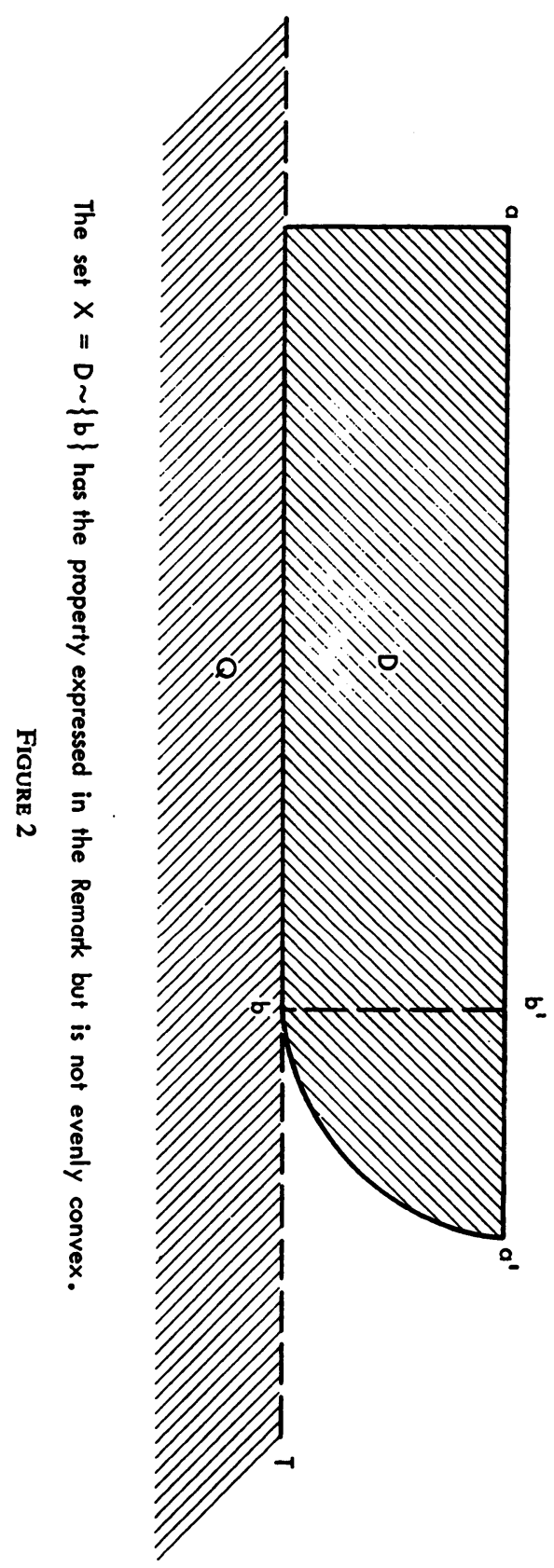


When the closure of $X$ is actually a polyhedron, the two properties are equivalent to the partial polyhedrality of $X$.

LEMMA 1. If $X$ and $Y$ are disjoint nonempty convex subsets of $E$ then $X$ is openly separated from $Y$ unless there is a point $p$ of $X$ which lies in every hyperplane separating $X$ from $Y$. Any such point $p$ satisfies at least one of the following conditions:

(1p) $p \in \operatorname{cl} Y$;

(2p) $p$ is an endpoint of a segment $[p, w]$ such that $w \in \operatorname{cl} Y$ and $[p, w] \subset(\operatorname{cl} X) \cap H$ for every hyperplane $H$ separating $X$ from $Y$;

(3p) there are sequences $p_{\alpha}$ in $E, x_{\alpha}$ in $X$, and $y_{\alpha}$ in $Y$ such that $p_{\alpha} \rightarrow p, y_{i} \in\left[p_{i}, x_{i}\right]$ for all $i$, and the sequence of segments $\left[p_{\alpha}, x_{\alpha}\right]$ converges to a ray which lies in $(\mathrm{cl} X) \cap H$ for every hyperplane $H$ separating $X$ from $Y$.

If $X$ and $Y$ are evenly convex then condition (3p) is satisfied for each point $p$ as described and each separating hyperplane $H$ such that the intersections $X \cap H$ and $Y \cap H$ are both closed and nonempty.

Proof. Let $F$ denote the set of all linear forms $f$ on $E$ such that $f(x) \leqq f(y)$ for all $x \in X$ and $y \in Y$. Since $F$ is nonempty, convex, and finite-dimensional, there is an element $g$ in the relative interior of $F$. Suppose there is no point $p$ as described, whence for each point $x$ of $X$ there exists $f_{x} \in F$ such that $f_{x}(x)<\inf f_{x} Y$. Note that $g$ lies in an open segment joining $f_{x}$ to another element $h_{x}$ of $F$ - say

$$
g=\lambda f_{x}+(1-\lambda) h_{x}, \quad \text { with } 0<\lambda<1 .
$$

For each $x \in X$ we have $f_{x}(x)<\inf f_{x} Y$ and $h_{x}(x) \leqq \inf h_{x} Y$, whence $g(x)<\inf g Y$. But then $X \cdot \mid Y$ by $g$ and the first assertion of Lemma 1 is proved.

Now suppose the point $p$ of $X$ lies in every hyperplane separating $X$ from $Y$, and for each positive integer $i$ let $N_{i}$ denote the (1/i)-neighborhood of $p$. The convex hull con $\left(X \cup N_{i}\right)$ is not separated from $Y$ and thus intersects $Y$, whence there are points $p_{i} \in N_{i}, x_{i} \in X$ and $y_{i} \in Y$ such that $y_{i} \in\left[p_{i}, x_{i}[\right.$. By choosing a subsequence if necessary, we may assume the sequence of segments $\left[p_{\alpha}, y_{\alpha}\right]$ is convergent to $\{p\}$, to a segment issuing from $p$, or to a ray issuing from $p$. These possibilities correspond to those listed in (1p), $(2 \mathrm{p})$ or $(3 \mathrm{p})$ respectively. To see that the segment or ray lies in every hyperplane $H$ separating $X$ from $Y$, note that the $x_{i}$ 's and $y_{i}$ 's are in opposite closed halfspaces bounded by $H$, while $\delta\left(\left\{p_{\alpha}\right\}, H\right) \rightarrow 0$.

Suppose, finally, that $X$ and $Y$ are evenly convex and $H$ is a separating hyperplane such that $X \cap H$ and $Y \cap H$ are both closed and nonempty. Choose $q \in Y \cap H$. If (lp) holds then $[q, p[\subset Y$ by the Remark and consequently $p \in X \cap Y$. If (2p) holds then $[p, w[\subset X$ and $[q, w[\subset Y$ by the Remark, whence $w \in X \cap Y$. These are contradictions completing the proof, for $X$ and $Y$ were assumed to be disjoint.

LEMMA 2. Suppose that $U$ and $V$ are disjoint nonempty evenly convex subsets of the Euclidean plane $P$, both intersecting a line $L$ which separates them. Suppose that the intersections $U \cap L$ and $V \cap L$ are separated in $L$ by a point $w$. 
(1) If $\delta(w,(U \cup V) \cap L)>0$ then $U$ and $V$ are strongly separated by a line through $w$. The same conclusion holds if $\delta(w, U \cup V)>0$, even when $U$ and $V$ are not evenly convex.

(2) If $w \notin U \cup V$ then $U$ and $V$ are strictly separated by a line through $w$.

(3) If $w \in V$ and $V$ is polyhedral at $w$ then $U$ is openly separated from $V$ by a line through $w$.

Proof. The set $P \sim L$ is the union of two open halfplanes $P_{U}$ and $P_{V}$ whose closures contain $U$ and $V$ respectively. The set $L \sim\{w\}$ is the union of two open rays $L_{U}$ and $L_{V}$ whose closures contain $U \cap L$ and $V \cap L$ respectively; in fact, $L_{U} \supset U \cap L$ because (under any of the three conditions) $w \notin U$. Since $w \notin U$ and $U$ is evenly convex (or perhaps, under (1), $u$ is not evenly convex but $\delta(w, U)>0$ ), the set

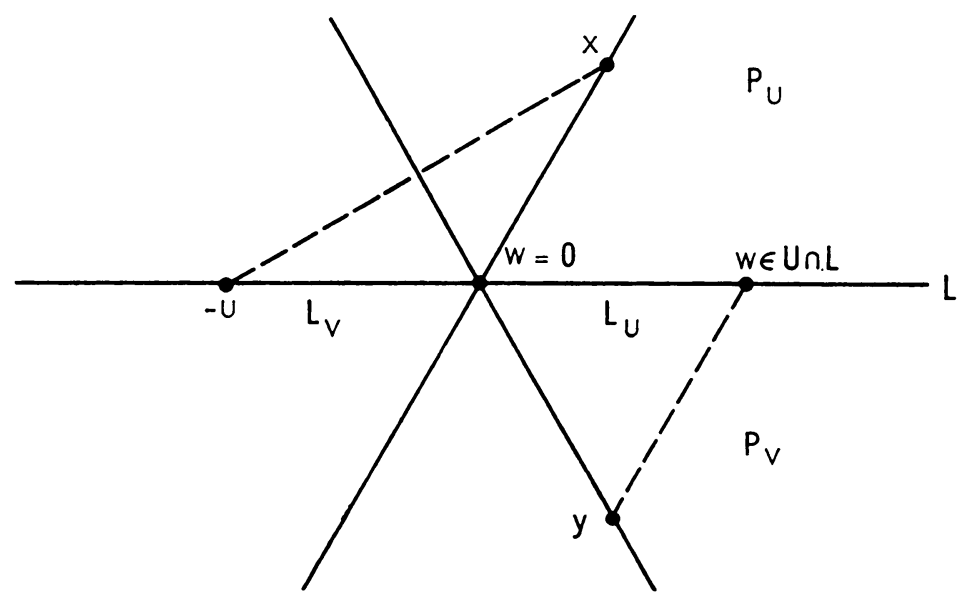

FIGURE 3

$P \sim U$ contains a line through $w$. Let us assume for notational simplicity that $w=0$, whence the line has the form $R x$ for some $x \in P_{U}$. Plainly $U$ is disjoint from every line of the form $R \bar{x}$ for $\bar{x} \in]-u, x$ [. (See Figure 3.)

Under (1) or (2), $w \notin V$ and $P \sim V$ contains a line through $w$. Plainly $V$ is disjoint from every line of the form $R \bar{y}$ for $\bar{y} \in] u, y[$. But for $\bar{y}$ in $] u, y[$ and sufficiently close to $u$, the line $R \bar{y}$ also has the form $R \bar{x}$ for $\bar{x} \in$ ] $-u, x$ [, and consequently $U$ and $V$ are strictly separated by $R \bar{y}$. It is also clear that the sets $U \sim W$ and $V \sim W$ are strongly separated by $R \bar{y}$ for each neighborhood $W$ of $w$, whence $U$ and $V$ are strongly separated by $R \bar{y}$ unless $w \in \operatorname{cl} U$ or $w \in \operatorname{cl} V$. The conditions $w \in \operatorname{cl} U$ and $w \in \mathrm{cl} V$ are explicitly excluded by the assumption, under (1), that $\delta(w, U \cup V)>0$; when $U$ and $V$ are evenly convex they are also excluded by the Remark in conjunction with the assumption that $\delta(w,(U \cup V) \cap L)>0$. 
Under (3) there is a point $y \in V \cap P_{V}$ such that $R \bar{y}$ supports $V$ for all $\bar{y} \in[u, y]$ and $V \cap R \bar{y}=\{0\}$ for all $\bar{y} \in] u, y[$. By choosing $\bar{y}$ sufficiently close to $u$ we obtain a line $R \bar{y}$ which openly separates $U$ from $V$ and which meets $V$ only at $w$.

Proofs of Theorems 4 and 5. We show first that the various conditions (e)-(q) imply $X \cdot \mid Y$ or $X \mid Y$, and then that the indicated $\cdot \mid$-pairs and $\mid$-pairs are maximal.

(e) and (k). By the standard separation theorem there is a hyperplane $H$ separating $X$ from $Y$. If $X$ is open, $X$ misses $H$ and $X \cdot \mid Y$. If $X$ is a strip, $H$ may be chosen as a supporting hyperplane of $X$, and $X$ misses $H$ (whence $X \cdot \mid Y$ ) or $X \supset H$ (whence $X \mid \cdot Y$ ).

(f), (h), (l) and (n). Supposing that $X$ is not openly separated from $Y$, let $p$ be as in Lemma 1 and let $H$ be a hyperplane separating $X$ and $Y$. Then of course $p \in X \cap H$ and $\delta(H, Y)=0$. If $Y$ misses $H$ then $X \mid Y$ and under (l) or (n) there is nothing to prove. If $Y$ misses $H$ under (f) then (lp) and (2p) are excluded by the fact that $Y$ is closed and thus ( $3 \mathrm{p}$ ) is satisfied. If $Y$ meets $H$ under (f), (h), (l) or (n) it follows from the last assertion of Lemma 1 that (3p) is satisfied. Note that $Y$ must meet $H$ under (h). Let the ray mentioned in (3p) be

$$
[p, p+u(=\{p+\lambda u: \lambda \geqq 0\}
$$

and note that, by the Remark, this ray is actually contained in $X$. Under (f) an immediate contradiction ensues. Under any of the remaining conditions (h), (l) and (n), (3p) is satisfied and the sets $X \cap H$ and $Y \cap H$ are closed and nonempty. For each point $q$ of $Y \cap H$ it follows from (3p) and the choice of $u$ that $[q, q+u($ $\subset \mathrm{cl} Y$ and then from the Remark that $[q, q+u(\subset Y \cap H$. Thus both $X \cap H$ and $Y \cap H$ are unions of translates of the ray [0, $u$. Choose $q_{0} \in Y \cap H$ and consider the two-dimensional flat $P$ determined by

$$
\left[p, p+u\left(\cup \left[q_{0}, q_{0}+u(.\right.\right.\right.
$$

Since $X$ and $Y$ are disjoint it is plain that $P$ contains boundary rays or asymptotes of both $X \cap H$ and $Y \cap H[2,1.2]$, and in each case the hypothesis of relative continuity is contradicted.

(g), (i), (m), (o), and (p). For each positive integer $d$ let $A_{d}$ denote one of the following assertions:

(g) implies $X \cdot \mid Y$

(i) implies $X \cdot \mid Y$

(m) implies $X \mid Y$

(o) implies $X \mid Y$

when $\operatorname{dim} E=d$ and $X$ and $Y$ are disjoint nonempty convex subsets of $E$.

(p) implies $X \mid Y$

The interpretation of $A_{d}$ is to be the same for all values of $d$, and the condition $((\mathrm{g}),(\mathrm{i}),(\mathrm{m}),(\mathrm{o})$ or $(\mathrm{p}))$ involved in this interpretation will be denoted by $(\gamma)$. Plainly $A_{1}$ is true and thus $A_{d}$ can be established for all values of $d$ by deriving $A_{d}$ from $A_{d-1}$. Suppose that $\operatorname{dim} E=d \geqq 2$. Let $X$ and $Y$ be disjoint nonempty convex 
subsets of $E$ satisfying $(\gamma)$ and let $H$ be a hyperplane separating $X$ from $Y$. If $X$ misses $H$ then $X \cdot \mid Y$, a desired conclusion. If $Y$ misses $H$ then $X \mid \cdot Y$, a desired conclusion when $(\gamma)$ is (m), (o) or (p). Further, if $Y$ misses $H$ and $(\gamma)$ is (g) or (i) then $\delta(H, Y)>0$ (for $Y$ admits no asymptote) and a translate of $H$ strongly separates $X$ from $Y$. There remains only the case in which the intersections $X \cap H$ and $Y \cap H$ are both nonempty. A routine verification shows that $(\gamma)$ is satisfied by the sets $X \cap H$ and $Y \cap H$, whence the inductive hypothesis $A_{d-1}$ guarantees the existence of a hyperplane $M$ in $H$ which (relative to $H$ ) separates $X \cap H$ from $Y \cap H$ in the appropriate manner. Under $(\mathrm{g})$ it is clear that $\delta(M, X)>0$, whence $X \cap H$ and $Y \cap H$ are strongly separated in $H$ by a translate of $M$. A similar situation prevails under (m), and also under (i) and (p) unless $M$ meets $Y$. Thus we may assume, replacing $M$ by a suitable translate, that the situation is as follows:

(g) or (m) $M$ misses $X \cup Y$

(i) or (p) $M$ meets $Y$ or misses $X \cup Y$.

Now choose $w \in M$, let $P$ be the (two-dimensional) orthogonal supplement of $M$ at $w$, and let $\pi$ denote the orthogonal projection of $E$ onto $P$. Let $U=\pi X$ and $V=\pi Y$. Then $\pi M=\{w\}, \pi H$ is a line $L$ through $w$, the sets $U$ and $V$ are separated in $P$ by $L$, and the intersections $U \cap L$ and $V \cap L$ are separated in $L$ by $w$. Further, the sets $U$ and $V$ are disjoint, evenly convex, and under certain conditions ((o) for $X$, (i), (o) and (p) for $Y$ ) are polyhedral at all of their points (use [5, 4.1]). Lemma 2 implies that $U$ and $V$ are separated in the appropriate manner by a line $T$ through $w$ in $P$, and then $\pi^{-1} T$ is a hyperplane which separates $X$ from $Y$ in the appropriate manner in $E$.

(j) and (q). Use the standard separation theorem and the relevant definitions.

We still must show that the $\cdot \mid$-pairs of Theorem 4 and the |-pairs of Theorem 5 are maximal. This follows from the observations below, showing first that the indicated pairs $(\mathscr{X}, \mathscr{Y})$ cannot be enlarged by adding to $\mathscr{X}$ and then that they cannot be enlarged by adding to $\mathscr{Y}$. Some of the details are left to the reader.

Let us suppose that $X$ is a proper convex subset of $E$.

(j) and (q). If (j) fails for $X$ then (by the definition of even convexity) $E \sim X$ contains a onepointed set $Y$ which is not closedly separated from $X$. Indeed, it is plain that $X$ is not nicely separated from $Y$.

(i) and (p). If (i) fails for $X$ then $E \sim X$ contains a flat $Y$ which is not closedly (and hence, also, not openly) separated from $X$.

(o). See the discussion of (o) for $Y$ below.

(h) and (n). If (h) fails for $X$ there are a supporting hyperplane $H$ of $X$ and a point $p$ of $H$ such that

$$
p \in \operatorname{cl}(X \cap H) \sim(X \cap H) .
$$

Then $E \sim X$ contains a compact Euclidean ball $Y$ which is tangent to $H$ at $p$, and $X$ is not nicely separated from $Y$.

(g) and (m). If (g) fails for $X$ there is a supporting hyperplane $H$ of $X$ which intersects $X$ and contains an asymptote $A$ of $X$. The set $E \sim X$ contains a compact 
Euclidean ball $B$ which is tangent to $H$ at a point of $A$. Let $Y$ denote the "spherical cylinder" cl con $(A \cup B)$. Then $Y$ admits no asymptote and $X$ is not nicely separated from $Y$.

(f). If (g) holds for $X$ but (f) fails for $X$ there is a supporting hyperplane $H$ of $X$ such that the intersection $X \cap H$ is unbounded and hence contains a ray [ $p, p+u$. There is a compact Euclidean ball $B$ which is separated from $X$ by $H$ and is tangent to $H$ at $p$. For notational simplicity we assume without loss of generality that $B$ is centered at the origin $O$. Let

$$
Y=\bigcup_{\lambda \geqq 1}\left(\lambda u+\frac{\lambda-1}{\lambda} B\right),
$$

a closed convex subset of $E \sim X$. Then $X$ is not openly separated from $Y$.

(l). If (m) holds for $X$ but (l) fails for $X$ there is a supporting hyperplane $H$ of $X$ such that the intersection $X \cap H$ admits a boundary ray [p,p+u( relative to $H$. Let $[q, q+u$ (be a parallel ray in $H \sim X$. Let $B$ be a compact Euclidean ball which is separated from $X$ by $H$ and is tangent to $H$ at the point $2 p-q$. Assume without loss of generality that $B$ is centered at $O$ and let

$$
Y=\operatorname{cl} \operatorname{con}\left(\left[q, q+u\left(\cup \bigcup_{\lambda \geqq 1}\left(\lambda u+\frac{\lambda-1}{\lambda}\right) B\right) .\right.\right.
$$

Then $Y$ is a closed convex subset of $E \sim X$ and $X$ is not nicely separated from $Y$. (Compare this with the example of Botts mentioned above.)

(e) and (k). If (e) fails for $X$ then $X$ intersects one of its supporting hyperplanes $H$. If $Y$ is an open halfspace which misses $X$ and is bounded by $H$, then $X$ is not openly separated from $Y$. If (k) fails for $X$ then $H$ can be chosen so that there exists $q \in H \sim X ; Y \cup\{q\}$ is a convex subset of $E \sim X$ and $X$ is not nicely separated from $Y \cup\{q\}$.

Now let us suppose that $Y$ is a proper convex subset of $E$.

(e) and (k). These place no restriction on $Y$.

(f). If (f) fails for $Y$ there is a onepointed set $X \subset(\mathrm{cl} Y) \sim Y$. The set $X$ is not openly separated from $Y$.

(l). If (l) fails for $Y$, refer to the above discussion of (h) for $X$.

(g). If (g) fails for $Y$ then $E \sim Y$ contains an asymptote $X$ of $Y$; $X$ is not openly separated from $Y$.

(m). If (m) fails for $Y$, refer to the above discussion of (g) for $X$.

(h) and (n). If (g) holds for $Y$ but (h) fails for $Y$, or if (m) holds for $Y$ but (n) fails for $Y$, then there is a supporting hyperplane $H$ of $Y$ such that the intersection $Y \cap H$ admits a boundary ray relative to $H$. Refer to the above discussion of (1) for $X$.

(i) and (o). If (g) holds for $Y$ but (i) fails for $Y$ there is a point $q$ of $Y$ at which $Y$ is not polyhedral. If (o) fails for $Y$ then $Y$ admits a projection which is not evenly convex (refer to the above discussion of (i) for $X$ ) or there is a point $q$ as described. 
With $C$ denoting the union of all rays which issue from $q$ and pass through the various points of $Y$, we conclude from $[5,3.3]$ that $C$ is not polyhedral and then from $[5,4.11]$ that there is a two-dimensional plane $P$ through $q$ such that the orthogonal projection $\pi C$ of $C$ onto $P$ is not closed. Let $T$ be an open ray issuing from $q$ such that

$$
T \subset \mathrm{cl} \pi C \sim \pi C
$$

and let $X=\pi^{-1} C$, an "open half-hyperplane" in $E$. Then $X$ is a partially polyhedral set in $E \sim Y$ but $X$ is not nicely separated from $C$ and hence also not from $Y$.

(p). If (o) holds for $Y$ but (p) fails for $Y$ then $Y$ admits an asymptote in a supporting hyperplane intersecting $Y$. Refer to the discussion of $(\mathrm{g})$ for $X$.

(j) and (q). If (i) holds for $Y$ but (j) fails for $Y$ then (p) holds for $Y$ but (q) fails for $Y$. Thus it suffices to show that in the latter instance $E \sim Y$ contains an evenly convex set $X$ which is not nicely separated from $Y$. As maximality is not claimed in the two-dimensional case, we may assume $\operatorname{dim} E=d \geqq 3$. Since $Y$ is not open there are supporting hyperplanes $H$ intersecting $Y$, and we want to produce one for which

$$
1 \leqq \operatorname{dim}(Y \cap H) \leqq d-2
$$

Note that for each supporting hyperplane $H$ intersecting $Y$, the intersection $Y \cap H$ is closed (for $Y$ admits no asymptote in $H$ ) and thus boundedly polyhedral (for $Y$ and hence $Y \cap H$ is polyhedral at each of its points); further, $Y \cap H \neq H$ because $Y$ is not a strip. Let $H_{0}$ be a supporting hyperplane for which the dimension $k$ of the intersection $Y \cap H_{0}$ is as large as possible. We claim $k \geqq 1$. Indeed, if $k=0$ then $Y \cap H_{0}$ consists of a single point $q$ but $Y \neq\{q\}$ because $Y$ is not onepointed. Since $Y$ is polyhedral at $q$ the relative boundary of $Y$ contains a segment issuing from $q$ and of course $Y$ is supported by a hyperplane containing this segment. Then the definition of $H_{0}$ is contradicted and we conclude that $k \geqq 1$. If $k \leqq d-2$ then $H_{0}$ has the desired property. If $k=d-1$ then (since $Y \cap H \neq H$ ) $Y \cap H$ has a face $F$ of dimension $d-2$ and it is easy (recalling that $Y$ is polyhedral at each of its points) to produce a supporting hyperplane $H$ for which $Y \cap H=F$. Henceforth $H$ will denote a supporting hyperplane for which (*) holds and $F$ will denote the intersection $Y \cap H$. We assume without loss of generality that $O$ is in the relative interior of $F$ and denote by $M$ the linear hull of $F$, by $L$ a line through $O$ in $M$, and by $P$ a two-dimensional linear subspace of the orthogonal supplement of $M$. Let $S$ denote the three-dimensional space $L \oplus P$ and let $Z$ denote the intersection $Y \cap S$.

There are a line $T$ through $O$ in $P$ and an open half-plane $Q$ bounded by $T$ such that $H \cap S=T \oplus L$ and $Z \subset(Q \cup\{0\}) \oplus L$. Let the set $D$ of Figure 2 be situated in $P$ with $b=0$ and with the relationship shown to $T$ and $Q$. Let $D^{\prime}$ be obtained from $D$ by rotating $D$ about the axis $\left[a, a^{\prime}\right]$ in $S$. Let $J$ be an open halfspace in $S$ such that $\left[b, b^{\prime}\right]$ lies in the boundary of $J$, half of $L$ is interior to $J$, and $J$ contains all points of $D$ to the left of $\left[b, b^{\prime}\right]$. Finally, let $X=D^{\prime} \cap J$. Then $X$ is evenly convex and is 
disjoint from $Z$, but $X$ is not nicely separated from $L$ in $S$. From this it follows that $X$ is not nicely separated from $Y$ in $E$ and thus the proof is complete.

Acknowledgements. Although this paper does not depend explicitly on any of T. S. Motzkin's work, it is closely related to some of his ideas. The property expressed in the Remark was introduced by him as Ganzseitigkeit $[8$, p. 16] and the partially polyhedral sets were called unrund $[8$, p. 20]. In 1951 he proposed to the author, in connection with [9], some separation problems for evenly convex sets.

R. T. Rockafellar kindly supplied a prepublication copy of [10].

\section{REFERENCES}

1. W. Fenchel, A remark on convex sets and polarity, Comm. Sém. Math. Univ. Lund (Medd. Lunds Univ. Math. Sem.) Tome Supplémentaire, 1952, 82-89.

2. D. Gale and V. Klee, Continuous convex sets, Math. Scand. 7 (1959), 379-391.

3. V. Klee, Convex sets in linear spaces, Duke Math. J. 18 (1951), 443-466.

4. - Strict separation of convex sets, Proc. Amer. Math. Soc. 7 (1956), 735-737.

5. - Some characterizations of convex polyhedra, Acta Math. 102 (1959), 79-107.

6. - Asymptotes and projections of convex sets, Math. Scand. 8 (1960), 356-362.

7. —, Asymptotes of convex bodies, Math. Scand. 15 (1967), 89-90.

8. Th. Motzkin, Beiträge zur Theorie der linearen Ungleichungen, Dissertation, Basel, 1936.

9. - Linear inequalities (mimeographed lecture notes), Univ. of California, Los Angeles, 1951.

10. R. T. Rockafellar, Polyhedral convex sets with some closed faces missing, (unpublished manuscript).

THE RAND CORPORATION,

Santa Monica, California

UNIVERSITY OF WASHINGTON,

Seattle, Washington 